\title{
Low-Dose Acetylsalicylic Acid Treatment Modulates the Production of Cytokines and Improves Trophoblast Function in an in Vitro Model of Early-Onset Preeclampsia
}

\author{
Shanika Panagodage, ${ }^{* \dagger}$ Hannah E.J. Yong, ${ }^{* \dagger}$ Fabricio Da Silva Costa, ${ }^{* \dagger}$ Anthony J. Borg, ${ }^{*}$ Bill Kalionis, ${ }^{* \dagger}$ \\ Shaun P. Brennecke, ${ }^{* \dagger}$ and Padma Murthi ${ }^{* \dagger \dagger}$
}

From the Department of Maternal-Fetal Medicine,* Pregnancy Research Centre, The Royal Women's Hospital, Parkville; the Department of Obstetrics and Gynaecology, ${ }^{\dagger}$ The University of Melbourne, Parkville; the Department of Medicine and the Department of Obstetrics and Gynaecology, ${ }^{\ddagger}$ School of Clinical Sciences, Monash University, and The Ritchie Centre, Monash Medical Centre, Clayton, Victoria, Australia

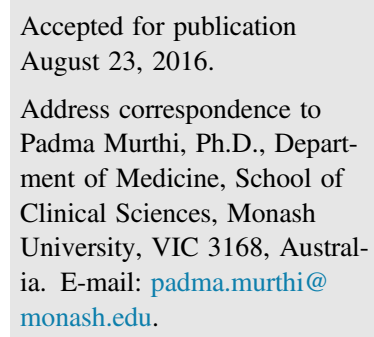

\begin{abstract}
Preeclampsia (PE), a serious hypertensive disorder of pregnancy, remains a leading cause of perinatal morbidity and mortality worldwide. Perturbed trophoblast function and impaired placental development early in pregnancy are key features. Low-dose acetylsalicylic acid (LDA) administered before 16 weeks' gestation significantly reduces the risk for PE. However, the exact mechanisms of action of LDA, particularly on trophoblast function, are unclear. We hypothesized that LDA influences placental trophoblast function and reverses PE-associated abnormalities. This study aimed to determine the effects of serum from normotensive women and from those with PE with or without LDA treatment on a model of placental syncytium. On cytokine profiling, LDA increased placental growth factor production and selectively restored PE serum-induced alterations in levels of cytokines [activated leukocyte cell adhesion molecule, CXCL-16, and ErbB3] to those in normotensive serum-treated cells. PE seruminduced increases in the apoptotic markers P53 mRNA expression, IKBKE mRNA expression, caspase 3 activity, and decreased BIRC8 mRNA expression, were attenuated by LDA treatment. LDA treatment also reduced abnormal differentiation caused by PE serum administration. Possible mechanisms by which LDA influences PE-affected trophoblast cells in vitro are by modulating cytokine secretion, reducing apoptosis to levels seen in normotensive serum-treated cells, and preventing the premature trophoblast differentiation commonly observed in PE. (Am J Pathol 2016, 186: 3217-3224; http://dx.doi.org/10.1016/ j.ajpath.2016.08.010)
\end{abstract}

Preeclampsia (PE) is a serious hypertensive disorder of pregnancy that affects about $3 \%$ of pregnancies worldwide and is a major cause of maternal and perinatal morbidity and mortality. ${ }^{1-3}$ The placenta is known to play a key role in $\mathrm{PE}$, with trophoblast dysfunction recognized as one of the major contributing factors. ${ }^{4}$ Various pharmacologic agents identified as candidate therapeutics for PE may have beneficial effects from influencing trophoblast function(s). These therapeutics include low-dose acetylsalicylic acid (LDA) or $\operatorname{aspirin}^{5}$; low-molecular-weight heparin ${ }^{6}$; folic acid $^{7}$; antioxidants such as vitamins $\mathrm{C}$ and $\mathrm{E}^{8}$; and calcium. ${ }^{9}$
Of these, prophylaxis with LDA has shown the greatest promise in reducing PE because of its lack of major side effects, ease of oral administration, and proven benefit especially when administered early in pregnancy. A metaanalysis showed a relative risk for PE of 0.47 in high-risk women whose LDA treatment was started at 16 weeks'

Supported by the Department of Maternal-Fetal Medicine, Pregnancy Research Centre, Royal Women's Hospital, Victoria, Australia.

S.P. and H.E.J.Y. contributed equally to this work.

Disclosures: None declared. 
gestation or earlier, whereas the benefit was not significant when treatment was started later. ${ }^{10}$ The reduction was even more marked in patients with severe PE, with a reported relative risk for severe PE of 0.18 in women who received LDA prophylaxis before 16 weeks' gestation, compared with those who had a commencement after 16 weeks. ${ }^{11}$ Nonetheless, while LDA shows promise as a potential PE therapeutic, the biological mechanisms underlying LDAmediated benefits remain elusive.

One such mechanism relates to the anticoagulant capacity of LDA. An ischemic placenta (consequent of PE) can give rise to vascular endothelial dysfunction, which can then lead to platelet activation and clotting. ${ }^{12}$ This process is preceded by excessive production of thromboxane, a vasoconstrictor that originates from platelets and stimulates platelet aggregation. ${ }^{13}$ LDA inhibits platelet thromboxane $\mathrm{A}_{2}$ biosynthesis, altering the imbalance between thromboxane $\mathrm{A}_{2}$ and prostacyclin, and is thus thought to favor the vasodilation that aids in lowering blood pressure and preventing PE. ${ }^{14}$ This mechanism, however, is too simplistic to explain the complete mechanism of action of LDA in preventing PE. A previous study by Bose et al, ${ }^{15}$ who treated trophoblast cells or villous explants with sera from women in the context of in vitro fertilization pregnancy success, demonstrated a potential role for LDA in modulating factors associated with trophoblast apoptosis. Further studies by Orendi et $\mathrm{al}^{16}$ and Han et $\mathrm{al}^{17}$ showed that LDA modulated the production of inflammatory cytokines when trophoblast cells were treated in the presence of anti-phospholipid antibodies. Nonetheless, the effects of LDA on trophoblast cytokine production and functions in a PE setting are yet to be investigated. ${ }^{4}$

Hence, in this study, we explored the functional effects of LDA on the trophoblast-derived cell line BeWo in a PE setting in vitro. This cell line is commonly used as a model of syncytiotrophoblasts of the placenta, as the cells secrete a diverse range of pregnancy hormones and proteins, and can also be induced in vitro to aggregate and fuse into a multinucleated syncytium. ${ }^{18}$ We hypothesized that LDA improves PE-affected trophoblast function by altering the production and secretion of cytokines, including placental growth factor (PIGF), with an overall beneficial effect on trophoblast cell function in vitro.

\section{Materials and Methods}

\section{Patient Sera}

Maternal serum was collected from women in the third trimester of pregnancy, with approval from the Human Research Ethics Committee at The Royal Women's Hospital (Parkville, VIC, Australia). Each woman provided written informed consent. PE was defined, according to the criteria from the Australasian Society for the Study of Hypertension in Pregnancy, ${ }^{19}$ as follows: a systolic pressure of $\geq 140$ $\mathrm{mmHg}$, a diastolic pressure of $\geq 90 \mathrm{mmHg}$, and a protein level of $\geq 0.3 \mathrm{~g}$ in a 24-hour urine collection or $\geq 2 \mathrm{~g}$ on a random urine dipstick test. Exclusion criteria were preexisting hypertension, multiple pregnancies, smoking, chemical dependency, diabetes mellitus, and systemic lupus erythematosus, which are factors known to predispose women to PE. Samples were collected from eight normotensive patients and four PE patients. None of the PE patients received LDA or other antihypertensive treatment before undergoing phlebotomy. Patients' characteristics are summarized in Table 1.

\section{Placental Growth Factor Cytokine Measurement}

PIGF concentrations in patient sera and cell cultureconditioned media were determined using fluorescence with the Delfia Xpress random-access platform immunoanalyzer (PerkinElmer, Waltham, MA) according to manufacturer's instructions. The Delfia Xpress PlGF kit (PerkinElmer) contained specific tracers and antibodies that enabled the quantitation of PlGF concentrations in picograms per milliliter. PlGF was measured to characterize patient sera and also was the first of the cytokines to be screened to determine the effects of LDA on trophoblast function.

\section{Trophoblast-Derived Cell Line}

The human choriocarcinoma-derived BeWo trophoblast cell line (clone B30) was a kind gift from Prof. Stephen Rogerson (The Royal Melbourne Hospital, Melbourne, VIC, Australia). Cells were cultured in RPMI 1640 medium; supplemented with $10 \%$ fetal calf serum, $200 \mathrm{U} / \mathrm{mL}$ penicillin, and $200 \mu \mathrm{g} / \mathrm{mL}$ streptomycin (Invitrogen, Carlsbad, $\mathrm{CA}$ ); and incubated at $37^{\circ} \mathrm{C}, 5 \% \mathrm{CO}_{2}$ and $95 \%$ air in a humidified chamber.

\section{Treatment of BeWo Cells}

Confluent cultures of $1 \times 10^{6}$ BeWo cells were serumstarved overnight in RPMI 1640 supplemented with $1 \%$ bovine serum albumin. The cells were then induced to aggregate and fuse with $20 \mu \mathrm{mol} / \mathrm{L}$ forskolin in RPMI 1640. With the exception of BeWo cells treated only with or without acetylsalicylic acid to obtain conditioned medium

Table 1 Clinical Characteristics of the Patient Samples in This Study

\begin{tabular}{lll}
\hline Patient characteristics* & $\begin{array}{l}\text { Normotensive } \\
(n=8)\end{array}$ & $\begin{array}{l}\text { Preeclampsia } \\
(n=4)\end{array}$ \\
\hline Gestational age at sampling, wk & $34.77 \pm 1.91$ & $33.90 \pm 2.52$ \\
Systolic blood pressure, $\mathrm{mmHg}$ & $<140$ & $162.5 \pm 8.5$ \\
Diastolic blood pressure, $\mathrm{mmHg}$ & $<90$ & $100.0 \pm 4.1$ \\
Proteinuria & None & $2+^{\dagger}$ \\
\hline
\end{tabular}

Data are expressed as means \pm SEM unless otherwise indicated.

${ }^{*} t$-test was used.

${ }^{\dagger}$ Value represents the the urine dipstick testing index. 
for PIGF measurements, all BeWo cells were also supplemented with $25 \%$ pooled maternal serum, which comprised either normotensive serum, PE serum alone, or PE serum with LDA. Acetylsalicylic acid (Sigma-Aldrich, Castle Hill, NSW, Australia) was prepared to a final concentration of $5 \mu \mathrm{g} / \mathrm{mL}$ in $95 \%$ ethanol as previously described by Bose et al. ${ }^{15}$ Experiments were performed in triplicate and repeated on six independent occasions.

\section{Cytokine Array}

The Human Cytokine Protein Array C8 (RayBiotech Inc., Norcross, GA) was used for cytokine profiling of BeWo cells. Conditioned medium was collected from BeWo cells after 72 hours of treatment with either normotensive serum, PE serum alone, or PE serum with LDA from six independent experiments. Conditioned media from each of the treatment groups were pooled and concentrated using Amicon 3000 concentrators (Merck Millipore, Billerica, MA). Cytokine profiles of each treatment group were then generated using the RayBio Human Cytokine Antibody Array C8 (RayBiotech Inc.) according to the manufacturer's instructions. The ECL-Western Chemiluminescence Detection kit (GE HealthCare, Amersham, UK) was used for detecting the immunoreactivity of 54 cytokines, according to the manufacturer's protocol. The ImageQuant LAS 4000 imager (GE HealthCare) was then used for imaging the cytokine array. Densitometric analysis was performed using Multi Gauge software version 3.0 (Fujifilm, Tokyo, Japan). Each immunoblot contained duplicate detection regions from each of the 54 target cytokines, and the mean of the measurements from each cytokine was calculated. Candidate cytokines were prioritized based on the greatest observed differences in immunoreactive signal intensities between treatment groups.

\section{RNA Extraction and cDNA Synthesis}

Total RNA from BeWo cells was extracted using the RNeasy MicroKit (Qiagen, Hilden, Germany) according to the manufacturer's instructions. cDNA was synthesized using $2 \mu \mathrm{g}$ of total cellular RNA as previously described. ${ }^{20}$

\section{Real-Time PCR}

Real-time PCR was performed on $12.5 \mathrm{ng} / \mu \mathrm{L} \mathrm{cDNA}$ in the Applied Biosystems 7500 PCR System (Foster City, CA) using TaqMan Gene Expression Assay FAM-labeled probes (CGB, Hs00361224_g1; P53, Hs00153349_m1) and VIC-labeled $18 S$ rRNA probe $(4319413 \mathrm{E}$; Thermo Fisher Scientific) as the housekeeping control gene. The reaction was performed initially for 10 minutes at $95^{\circ} \mathrm{C}$, then 15 seconds at $95^{\circ} \mathrm{C}$, and 1 minute at $60^{\circ} \mathrm{C}$ for 40 cycles as previously described. ${ }^{20}$ The relative gene expression was determined according to the $2^{-\Delta \Delta C T}$ method. ${ }^{21}$

\section{Enzyme-Linked Immunosorbent Assay}

An enzyme-linked immunosorbent assay was used for measuring CXCL-16 protein levels of serum-treated BeWo cells with and without acetylsalicylic acid. The kit was purchased from Proteintech Group (Rosemont, IL). Experiments were performed on cellular extracts using a 1:20 dilution, according to the manufacturer's protocol.

\section{Cellular Apoptosis Array}

The Cellular Apoptosis TaqMan PCR array (Applied Biosystems) for gene profiling was used for screening for genes that showed differential expression in BeWo cells treated with normotensive serum, PE serum, and PE serum with LDA. The array plate contained 84 gene-specific primer sets, with a panel of five housekeeping-gene primers for normalization. The housekeeping genes consisted of $18 S$ rRNA, $\beta_{2}$-microglobulin $(B 2 M)$, hypoxanthine phosphoribosyltransferase 1 (HPRT1), glyceraldehyde-3-phosphate dehydrogenase $(G A P D H)$, and $\beta$-actin $(A C T B)$. The PCR reaction was performed in an Applied Biosystems 7500 PCR System using the same cycling parameters as for the real-time PCR. Data $\left(C_{t}\right.$ values) were analyzed using the ABI Sequence Detector System software version 2.0 (Thermo Fisher Scientific). The relative gene expression values, or fold changes, were analyzed using the DataAssist Software version 3.0 (Applied Biosystems/Thermo Fisher Scientific). Candidate genes were prioritized based on the differences in gene mRNA expression in cells treated with PE serum plus LDA compared with those treated with PE serum alone or normotensive serum.

\section{Caspase 3 Activity Assay}

Caspase 3 activity was measured in BeWo cells using the ApoAlert Caspase Colorimetric assay kit (Clontech Laboratories Inc., Mountain View, CA) specific for the caspase 3 activity according to the manufacturer's instructions. Chromogen absorbance was measured at $405 \mathrm{~nm}$ using a SpectraMax Plus microplate reader (Molecular Devices Corp., Sunnyvale, CA).

\section{Functional Analysis of BeWo Cells Using the xCELLigence System}

The BeWo cell differentiation profiles of each treatment group were monitored for 72 hours using the E-Plate 16 (Acea Biosciences, San Diego, CA) in the real-time electrical impedance-based xCELLigence System (Acea Biosciences), according to the manufacturer's protocol. Data are expressed as arbitrary cell indexes. The experimental results were analyzed as previously described. ${ }^{22}$

\section{Statistical Analysis}

The $t$-test, one-way analysis of variance with Bonferroni post-test, and $2 \times 2$ contingency table with Fisher exact test were used where appropriate. All analyses were performed 
on GraphPad Prism software version 5.02 (GraphPad Software Inc., La Jolla, CA). The data are expressed as means \pm SEM. A $P$ value of $<0.05$ was regarded as statistically significant.

\section{Results}

\section{Patient Characteristics}

The difference in gestational age at sampling between the normotensive and PE patients was not significant. PE patients had severe hypertension and proteinuria that met the study criteria for the diagnosis of PE.

\section{PIGF Concentrations in Sera and Cell Culture-Conditioned Media}

PlGF cytokine concentrations were first determined in patient sera and cell culture-conditioned media. PE patients had, on average, significantly reduced (by 4.4-fold) PIGF concentrations compared with those in the normotensive patients (Figure 1A). Analysis of the effect of LDA on BeWo trophoblast PIGF production was performed in the absence of sera, as maternal sera contains PIGF, which would have confounded PIGF measurements after treatment. LDA treatment significantly increased (by 1.5 -fold) BeWo trophoblast production of PIGF compared with that in the vehicle control (Figure 1B). Subsequent in vitro experiments were performed in the presence of sera.

\section{Cytokine Profiling}

Conditioned media from each treatment group were assessed for cytokine secretion after treatment of BeWo cells with maternal sera for 72 hours. Immunoblots were quantitated to determine the signal intensities of cytokines. Candidates were selected by the identification of cytokines that were substantially altered by treatment with PE serum compared with normotensive serum and that were restored by treatment with LDA. Of the 54 cytokines screened in the array, the most promising candidates were activated leukocyte cell adhesion molecule (ALCAM), CXCL-16, and ErbB3 (Figure 2A). CXCL-16 was prioritized for further validation, given its restoration by LDA to levels closest to those seen in cells treated with normotensive serum; because it is a known regulator of trophoblast invasion ${ }^{23,24}$; and because there is an association between abnormal, shallow trophoblast invasion and PE. ${ }^{4}$ CXCL-16 mRNA expression was significantly decreased in BeWo cells treated with PE serum alone compared with that in BeWo cells treated with normotensive serum (Figure 2B). This decrease was significantly attenuated by LDA, with $C X C L-16$ mRNA expression in BeWo cells treated with PE serum with LDA restored to levels near those observed in BeWo cells treated with normotensive serum (Figure 2B). Similar effects were also observed at the protein level (Figure 2C).
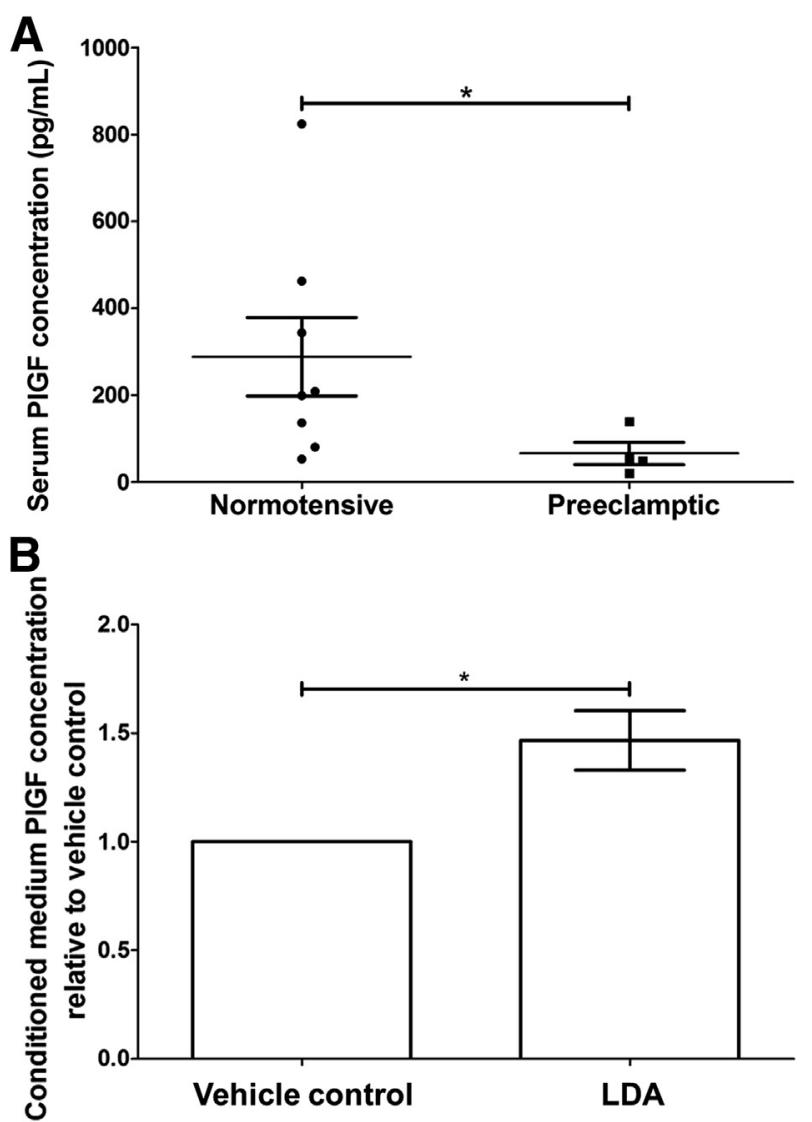

Figure 1 Placental growth factor (PIGF) concentrations in serum from normotensive and preeclamptic (PE) women, and its modulation by lowdose acetylsalicylic acid (LDA) of trophoblast cell secretions in vitro. PIGF concentrations in serum and cell culture-conditioned media, as measured using a Delfia Xpress random-access platform immunoanalyzer (PerkinElmer, Waltham, MA). A: PIGF concentrations are significantly lower in PE serum compared with normotensive serum. B: Treatment with LDA improved PIGF secretion by BeWo trophoblast cells. Data are expressed as means \pm SEM. $n=4$ (A, preeclamptic, and $\mathbf{B}$, both groups); $n=8$ (A, normotensive). ${ }^{*} P<0.05$ (t-test).

\section{BeWo Cell Apoptosis}

The effect of LDA treatment on BeWo apoptosis was determined by measurement of the expression of apoptotic markers using real-time PCR, an apoptotic pathway cDNA array, and an assay for caspase 3 activity. Figure $3 \mathrm{~A}$ depicts P53 mRNA expression relative to that of $18 S$ rRNA in cells treated with normotensive serum, PE serum alone, and PE serum plus LDA. Compared with cells treated with normotensive serum, there was a significant increase (of $>20$-fold) in P53 mRNA expression relative to $18 \mathrm{~S} \mathrm{rRNA}$ in BeWo cells treated with PE serum alone. However, with the addition of LDA, the BeWo cells showed significantly reduced, by 20.0-fold, P53 mRNA levels compared with the BeWo cells treated with PE serum alone. There was no significant difference in P53 mRNA relative to $18 S$ rRNA between normotensive cells and those treated with PE plus LDA.

Further screening for apoptotic markers using the apoptotic pathway cDNA array demonstrated a 4.0 -fold 

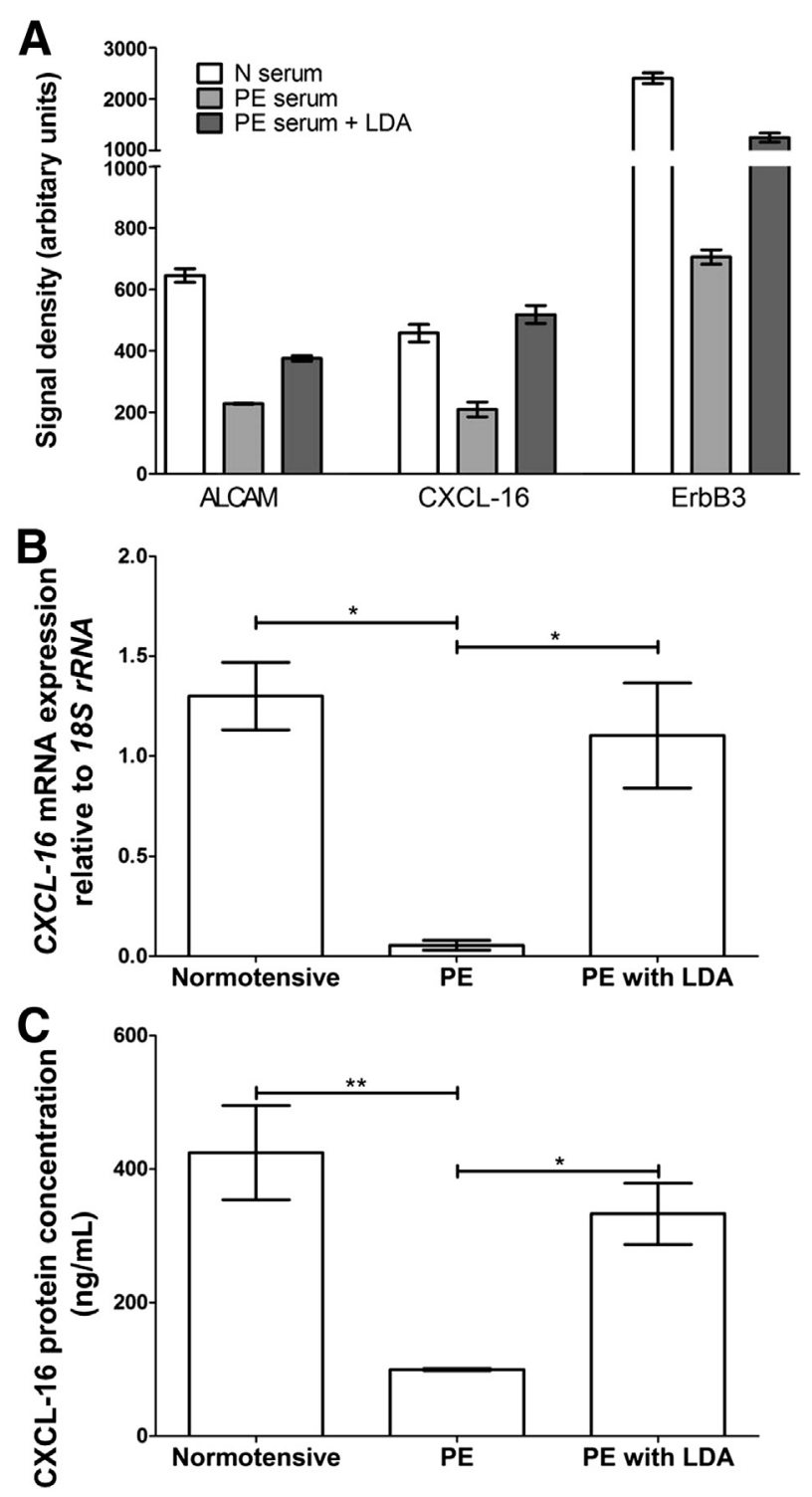

Figure 2 Effects of low-dose acetylsalicylic acid (LDA) on cytokine production. Cytokines secreted by BeWo trophoblast cells, as screened with a cytokine array. A: The protein expression levels of candidate cytokines of interest, activated leukocyte cell adhesion molecule (ALCAM), CXCL-16, and ErbB3 are decreased in cells treated with preeclamptic (PE) serum and are enhanced by LDA treatment to near the levels observed in cells treated with normotensive (N) serum. B and C: CXCL-16, selected for further validation using real-time PCR (B) and enzyme-linked immunosorbent assay (C), confirms changes observed in the screening. Data are expressed as means \pm SEM. $n \geq 3$ independent experiments $(\mathbf{A}-\mathbf{C})$. ${ }^{*} P<0.05$, ${ }^{* *} P<0.01$ (one-way analysis of variance with Bonferroni post-test).

increase in IKBKE mRNA expression and a 2.0-fold decrease in BIRC8 mRNA expression in cells treated with PE serum alone compared with cells treated with normotensive serum. However, in BeWo cells treated with PE serum with LDA, a 20.0-fold decrease in IKBKE and a 4.0-fold increase in BIRC8 were observed compared with that in cells treated with PE serum alone. Validation by realtime PCR demonstrated a significant increase (of 2.6-fold) in IKBKE in cells treated with PE serum alone compared with those treated with normotensive serum; this value was significantly decreased, by 3.1 -fold, in cells treated in PE serum with LDA (Figure 3B). mRNA expression of BIRC8 was significantly decreased, by 2.6 -fold, in cells treated with PE serum alone compared with those treated with normotensive serum; this value was significantly increased, by 2.8 -fold, in cells treated with PE serum with LDA (Figure 3C).

A 4.0-fold increase in caspase 3 activity was observed in BeWo cells treated with PE serum alone compared with that in those treated with normotensive serum (Figure 3D). However, in cells treated with PE serum with LDA, caspase 3 activity was significantly decreased (halved) compared with that in cells treated with PE serum alone (Figure 3D).

\section{BeWo Cell Differentiation}

Differentiation of BeWo cells was measured using real-time PCR analysis of the differentiation marker $C G B$ and functional xCELLigence assays. As shown in Figure 4A, CGB mRNA expression relative to that of $18 S$ rRNA was significantly increased, by 13.0 -fold, in cells treated with PE serum alone compared to normotensive serum-treated cells. However, in the presence of PE serum with LDA, CGB mRNA expression was significantly reduced, by 13.5 -fold, compared with that in the BeWo cells treated with PE serum alone. No significant difference in $C G B$ mRNA expression was observed between the cells treated with normotensive serum and those treated with PE serum with LDA.

To further investigate the effects of the different maternal sera and LDA treatment on BeWo cell differentiation/ fusion, the xCELLigence system was used. Different cell aggregation and fusion profiles were observed in each treatment group (Figure 4B). At 24 hours, the cell indexes of cells treated with either PE serum alone or PE serum plus LDA were significantly higher, by 2.0 -fold and 1.8 , respectively, than that of cells treated with normotensive serum (Figure 4C). However, by 72 hours, while there was still a significant increase of 1.7-fold in the cell index of cells treated with PE serum alone, the cell index of cells treated with PE serum with LDA was not significantly different compared with that of normotensive serumtreated cells (Figure 4D).

\section{Discussion}

Our study has demonstrated the influence of LDA on BeWo cell function in the context of PE. We confirm that the cytokine PlGF is significantly decreased in PE serum compared with that in normotensive serum and demonstrate that LDA can increase trophoblast secretion of PlGF. LDA also restored abnormal trophoblast production of additional cytokines induced by PE serum to levels observed in normotensive serum, and attenuated abnormal trophoblast apoptosis and growth caused by PE serum. Therefore, this study provides supporting evidence of the functional 

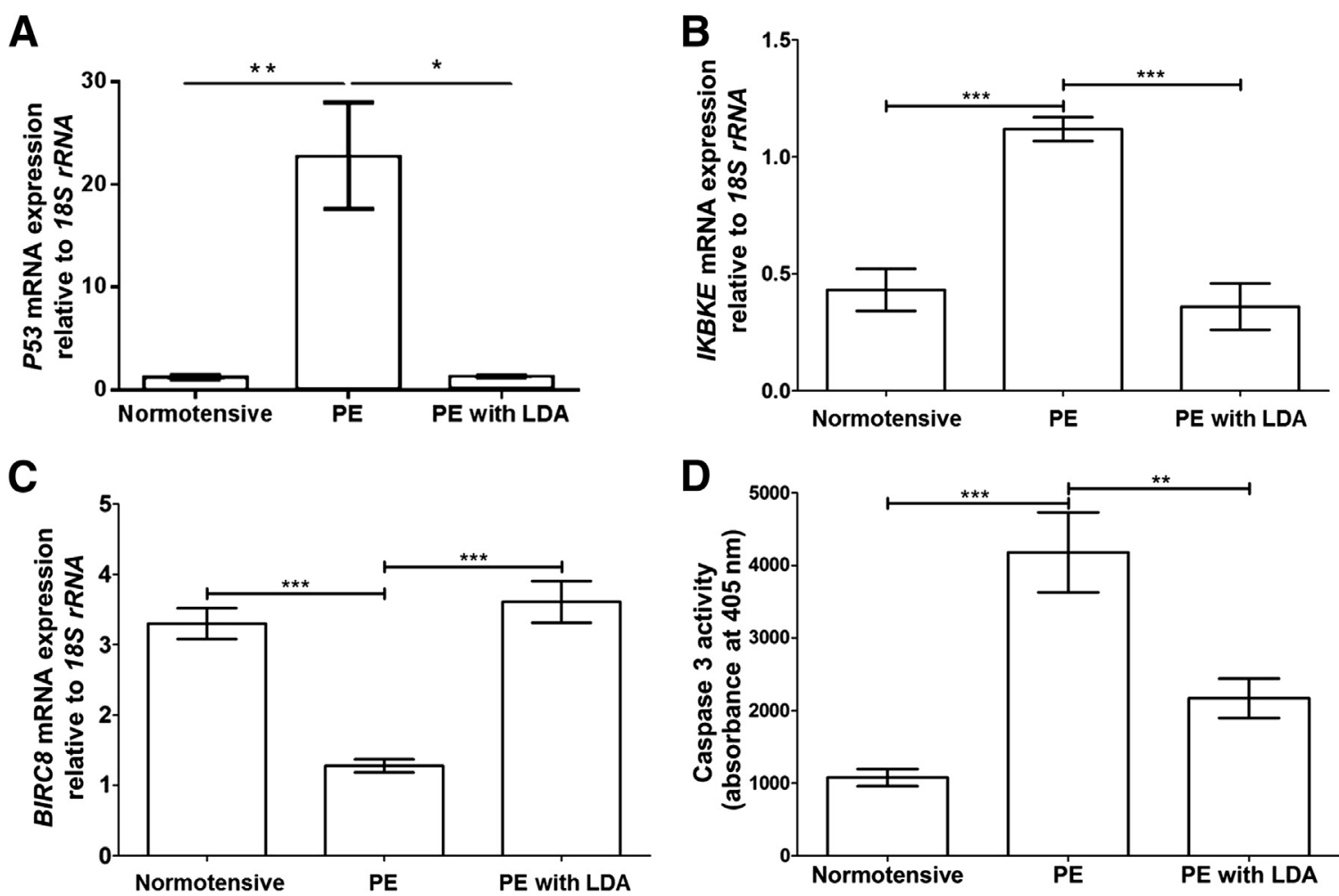

Figure 3 Effect of low-dose acetylsalicylic acid (LDA) on apoptosis in BeWo cells. Apoptosis, as determined by changes in mRNA expression of multiple apoptotic markers, P53 (A), IKBKE (B), and BIRC8 (C), and caspase 3 activity (D), using real-time PCR analysis and a colorimetric assay, respectively. Preeclamptic (PE) serum alone significantly increases P53 and IKBKE mRNA expression, reduces BIRC8 mRNA expression, and enhances caspase 3 activity in BeWo trophoblasts above that in normotensive serum-treated cells. These effects induced by PE serum are all attenuated by the addition of LDA. Data are expressed as means \pm SEM. $n \geq 3$ independent experiments (A-D). ${ }^{*} P<0.05$, ${ }^{* *} P<0.01$, and ${ }^{* * *} P<0.001$ (one-way analysis of variance with Bonferroni post-test).

mechanisms of cytokine production, apoptosis, and differentiation through which LDA may modulate the risk for PE.

The lower cytokine concentrations of PlGF in sera from PE women were consistent with those from past studies. ${ }^{25-27}$ Reduced serum PIGF concentrations are detectable early in pregnancy, even before the clinical features of hypertension and proteinuria develop, and given the significance of PIGF as a predictive biomarker of $\mathrm{PE},{ }^{28,29}$ we decided to first ascertain whether LDA had any beneficial effect of placental trophoblast PIGF production. Placental production of PIGF is correlated with maternal serum concentrations. ${ }^{30}$ The concentration of LDA chosen for this study reflects physiologic levels during treatment in vivo. ${ }^{15}$ As maternal sera also contained PIGF and thus would have confounded the measurements obtained, sera were omitted in the treatment of BeWo trophoblast cells with acetylsalicylic acid. Given the promising result of LDA increasing trophoblast PlGF cytokine production, we expanded the study further by screening additional cytokines and performing several functional assays in vitro in the presence of maternal sera.

Cytokine profiling in our study demonstrated decreases in the levels of secretion of ALCAM, CXCL-16, and ErbB3 into BeWo cells conditioned with PE serum alone; these levels were restored near to those seen in cells treated with normotensive serum when LDA was added in vitro.
ALCAM is an adhesion molecule involved in blastocyst implantation into the maternal uterus and facilitates maternal-fetal communication. ${ }^{31,32}$ ALCAM is also expressed by placental mesenchymal stem cells and is involved in the placental differentiation process. ${ }^{33} \mathrm{CXCL}$ 16 promotes proliferation and invasion of first-trimester trophoblast cells into the decidua, as well as recruitment of maternal lymphocytes into the maternal-fetal interface. ${ }^{23,24}$ ErbB3 is implicated in regulating differentiation. ${ }^{34}$ $E R B B 3$ (alias HER3), the gene encoding for the epidermal growth factor receptor, is expressed in terminally differentiated and apoptotic mammary epithelial cells. ${ }^{34}$ In rabbit placenta it was demonstrated that ErbB3 was expressed in cytotrophoblasts, as well as syncytiotrophoblasts, and is involved in the process of decidualization. ${ }^{35}$ However, the role of ErbB3 in human trophoblasts is largely unknown. Thus, cytokine profiling in trophoblasts suggests that LDA has a restorative effect on the trophoblast production of these candidate placental cytokines and requires further investigation. Using maternal serum from a gestational age-matched set of PE-affected and normotensive (control) women, the present study provides evidence that LDA treatment alters cytokine production by trophoblasts exposed to PE serum. These data suggest that LDA may counterbalance abnormal cytokine production in PEaffected BeWo cells and potentially contribute to restoring 
A
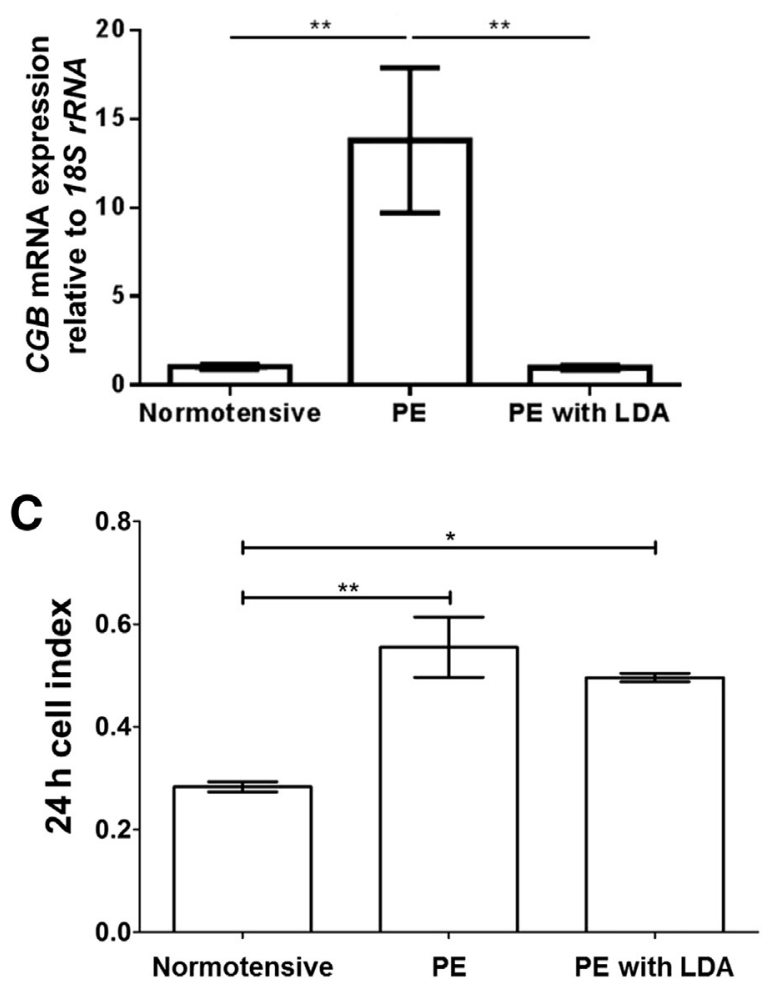

B
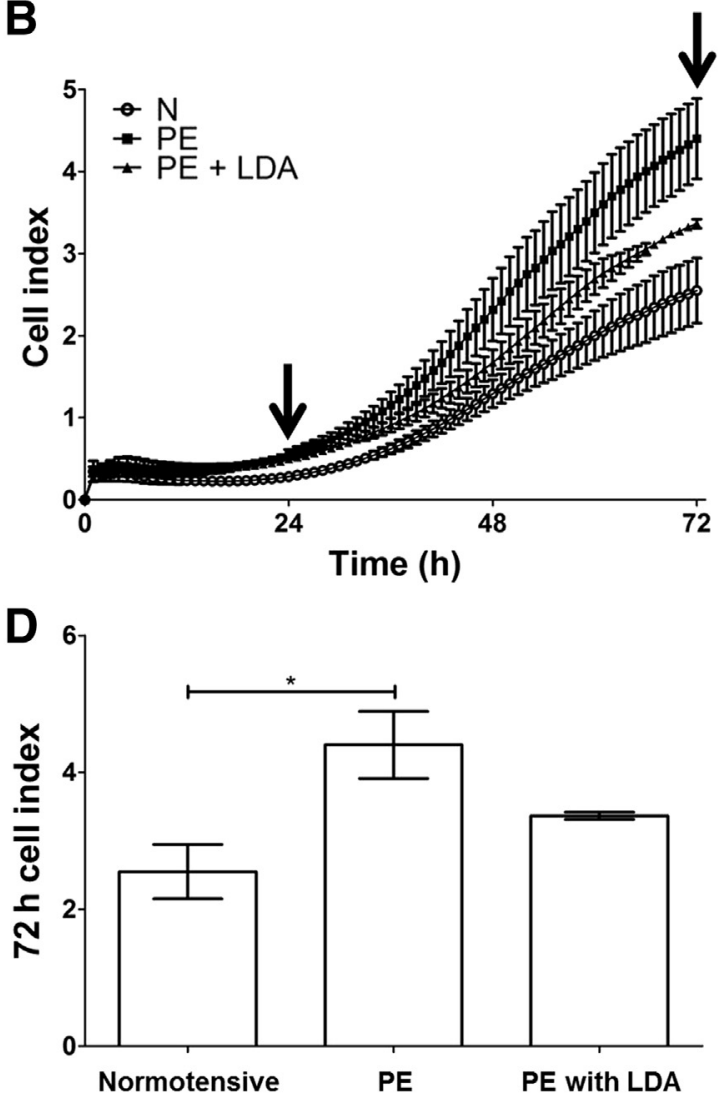

Figure 4 Effect of low-dose acetylsalicylic acid (LDA) on BeWo cell differentiation. A: Molecular differentiation, as determined by mRNA expression of CGB relative to $18 \mathrm{~S} r R N A$, which is modulated by LDA treatment. B: Cell growth and fusion in BeWo cells treated with serum with/without LDA, as measured by xCELLigence assay (ACEA Biosciences, San Diego, CA) over 72 hours. Arrows indicate time points of interest that were analyzed in greater detail. C and D: Preeclamptic (PE) serum-treated cells (with or without LDA) have increased growth at 24 hours (C), which is attenuated by LDA at 72 hours compared with that in normotensive $(\mathrm{N})$ serum-treated cells $(\mathbf{D})$. Data are expressed as means \pm SEM. $n \geq 3$ independent experiments $(\mathbf{A}-\mathbf{D})$. ${ }^{*} P<0.05$, ${ }^{* * P}<0.01$ (oneway analysis of variance with Bonferroni post-test).

placental function in PE. Further understanding of ALCAM-, CXCL-16-, and ErbB3-mediated molecular mechanisms in PE may provide additional insights into the pathogenesis of PE and present novel diagnostic and therapeutic targets in PE-affected pregnancies.

Increased apoptosis is commonly observed in PE. ${ }^{36}$ In the present study, evidence of increased trophoblast apoptosis was observed through significantly increased mRNA expression of the apoptotic markers P53 and IKBKE, reduced BIRC8 mRNA expression, and increased caspase 3 activity in BeWo cells treated with PE serum compared with those in cells treated with normotensive serum. The significant reduction in P53 and IKBKE mRNA expression, increased BIRC8 mRNA expression, and decreased caspase 3 activity in cells treated with PE serum with LDA suggest that LDA may have an anti-apoptotic effect on these cells and that LDA may directly or indirectly control the apoptotic pathway in trophoblasts. This finding is similar to those from a study by Bose et al, ${ }^{15}$ in which BeWo cell apoptosis was increased in the presence of sera collected from women with in vitro fertilization failure; this increase was reversed with the addition of LDA in vitro.
The effect of acetylsalicylic acid was further assessed using a real-time functional xCELLigence assay. Forskolininduced BeWo cell growth is characterized by aggregation of cells and fusion into multinucleated syncytium within 72 hours. At 72 hours after $5-\mu \mathrm{g} / \mathrm{mL}$ acetylsalicylic acid treatment, BeWo cells cultured in PE serum with LDA showed reduced aggregation and fusion compared with those in cells treated with PE serum alone, indicating that acetylsalicylic acid plays a role in regulating cell aggregation and fusion. The effect of acetylsalicylic acid on restoring trophoblast function is a gradual, time-dependent process. The restorative effects were observed only after 72 hours in cells treated with PE serum plus LDA, and were not apparent at the earlier time point of 24 hours.

In conclusion, our study is the first to demonstrate the restorative potential of LDA treatment in vitro. This restorative potential may improve defective trophoblast syncytialization in PE by altering the production of specific cytokines, decreasing apoptosis, and altering cell aggregation and fusion. Our findings provide evidence about novel mechanisms by which LDA may work as a clinical prophylaxis against PE in early pregnancy. Further 
understanding of these mechanisms will aid in the development of new diagnostic and therapeutic targets.

\section{References}

1. Duley L, Henderson-Smart DJ, Meher S, King JF: Antiplatelet agents for preventing pre-eclampsia and its complications. Cochrane Database Syst Rev 2007:CD004659

2. Thornton C, Dahlen H, Korda A, Hennessy A: The incidence of preeclampsia and eclampsia and associated maternal mortality in Australia from population-linked datasets: 2000-2008. Am J Obstet Gynecol 2013, 208:476e1-476e5

3. WHO: The World Health Report 2005 - make every mother and child count. Geneva, Switzerland: World Health Organization, 2005

4. Eastabrook G, Brown M, Sargent I: The origins and end-organ consequence of pre-eclampsia. Best Pract Res Clin Obstet Gynaecol 2011, 25:435-447

5. Bujold E, Morency AM, Roberge S, Lacasse Y, Forest JC, Giguere Y: Acetylsalicylic acid for the prevention of preeclampsia and intrauterine growth restriction in women with abnormal uterine artery Doppler: a systematic review and meta-analysis. J Obstet Gynaecol Can 2009, 31:818-826

6. Mello G, Parretti E, Fatini C, Riviello C, Gensini F, Marchionni M, Scarselli GF, Gensini GF, Abbate R: Low-molecular-weight heparin lowers the recurrence rate of preeclampsia and restores the physiological vascular changes in angiotensin-converting enzyme DD women. Hypertension 2005, 45:86-91

7. Wen SW, Chen XK, Rodger M, White RR, Yang Q, Smith GN, Sigal RJ, Perkins SL, Walker MC: Folic acid supplementation in early second trimester and the risk of preeclampsia. Am J Obstet Gynecol 2008, 198:45e1-45e7

8. Kharb S: Vitamin E and C in preeclampsia. Eur J Obstet Gynecol Reprod Biol 2000, 93:37-39

9. Astrup A: Calcium reduces risk of pre-eclampsia. Lancet 2010, 376: 1986-1987

10. Bujold E, Roberge S, Lacasse Y, Bureau M, Audibert F, Marcoux S, Forest JC, Giguere Y: Prevention of preeclampsia and intrauterine growth restriction with aspirin started in early pregnancy: a metaanalysis. Obstet Gynecol 2010, 116:402-414

11. Roberge S, Nicolaides KH, Demers S, Villa P, Bujold E: Prevention of perinatal death and adverse perinatal outcome using low-dose aspirin: a meta-analysis. Ultrasound Obstet Gynecol 2013, 41:491-499

12. Janes SL, Kyle PM, Redman C, Goodall AH: Flow cytometric detection of activated platelets in pregnant women prior to the development of pre-eclampsia. Thromb Haemost 1995, 74:1059-1063

13. Thorp JA, Walsh SW, Brath PC: Low-dose aspirin inhibits thromboxane, but not prostacyclin, production by human placental arteries. Am J Obstet Gynecol 1988, 159:1381-1384

14. Wallenburg HC, Dekker GA, Makovitz JW, Rotmans P: Low-dose aspirin prevents pregnancy-induced hypertension and pre-eclampsia in angiotensin-sensitive primigravidae. Lancet 1986, 1:1-3

15. Bose P, Black S, Kadyrov M, Weissenborn U, Neulen J, Regan L, Huppertz B: Heparin and aspirin attenuate placental apoptosis in vitro: implications for early pregnancy failure. Am J Obstet Gynecol 2005, 192:23-30

16. Orendi K, Gauster M, Moser G, Meiri H, Huppertz B: Effects of vitamins $\mathrm{C}$ and $\mathrm{E}$, acetylsalicylic acid and heparin on fusion, beta-hCG and PP13 expression in BeWo cells. Placenta 2010, 31:431-438

17. Han CS, Mulla MJ, Brosens JJ, Chamley LW, Paidas MJ, Lockwood CJ, Abrahams VM: Aspirin and heparin effect on basal and antiphospholipid antibody modulation of trophoblast function. Obstet Gynecol 2011, 118:1021-1028

18. Pattillo RA, Gey GO: The establishment of a cell line of human hormone-synthesizing trophoblastic cells in vitro. Cancer Res 1968, 28:1231-1236
19. Brown MA, Hague WM, Higgins J, Lowe S, McCowan L, Oats J, Peek MJ, Rowan JA, Walters BN; Australasian Society for the Study of Hypertension in P: The detection, investigation and management of hypertension in pregnancy: executive summary. Aust $\mathrm{N} \mathrm{Z} \mathrm{J} \mathrm{Obstet}$ Gynaecol 2000, 40:133-138

20. Borg AJ, Yong HE, Lappas M, Degrelle SA, Keogh RJ, Da SilvaCosta F, Fournier T, Abumaree M, Keelan JA, Kalionis B, Murthi P: Decreased STAT3 in human idiopathic fetal growth restriction contributes to trophoblast dysfunction. Reproduction 2015, 149:523-532

21. Livak KJ, Schmittgen TD: Analysis of relative gene expression data using real-time quantitative PCR and the 2(-Delta C(T)) method. Methods 2001, 25:402-408

22. Yong HEJ, Murthi $\mathrm{P}$, Wong $\mathrm{MH}$, Kalionis $\mathrm{B}$, Cartwright JE, Brennecke SP, Keogh RJ: Effects of normal and high circulating concentrations of activin A on vascular endothelial cell functions and vasoactive factor production. Pregnancy Hypertens 2015, 5:346-353

23. Huang Y, Zhu XY, Du MR, Li DJ: Human trophoblasts recruited T lymphocytes and monocytes into decidua by secretion of chemokine CXCL16 and interaction with CXCR6 in the first-trimester pregnancy. J Immunol 2008, 180:2367-2375

24. Huang Y, Zhu XY, Du MR, Wu X, Wang MY, Li DJ: Chemokine CXCL16, a scavenger receptor, induces proliferation and invasion of first-trimester human trophoblast cells in an autocrine manner. Hum Reprod 2006, 21:1083-1091

25. Livingston JC, Chin R, Haddad B, McKinney ET, Ahokas R, Sibai BM: Reductions of vascular endothelial growth factor and placental growth factor concentrations in severe preeclampsia. Am J Obstet Gynecol 2000, 183:1554-1557

26. Robinson CJ, Johnson DD, Chang EY, Armstrong DM, Wang W: Evaluation of placenta growth factor and soluble Fms-like tyrosine kinase 1 receptor levels in mild and severe preeclampsia. Am J Obstet Gynecol 2006, 195:255-259

27. Torry DS, Wang HS, Wang TH, Caudle MR, Torry RJ: Preeclampsia is associated with reduced serum levels of placenta growth factor. Am J Obstet Gynecol 1998, 179:1539-1544

28. Liu Y, Zhao Y, Yu A, Zhao B, Gao Y, Niu H: Diagnostic accuracy of the soluble Fms-like tyrosine kinase-1/placental growth factor ratio for preeclampsia: a meta-analysis based on 20 studies. Arch Gynecol Obstet 2015, 292:507-518

29. Poon LC, Nicolaides KH: Early prediction of preeclampsia. Obstet Gynecol Int 2014, 2014:297397

30. Weed S, Bastek JA, Anton L, Elovitz MA, Parry S, Srinivas SK: Examining the correlation between placental and serum placenta growth factor in preeclampsia. Am J Obstet Gynecol 2012, 207: $140 \mathrm{e} 1-140 \mathrm{e} 6$

31. Fujiwara H, Tatsumi K, Kosaka K, Sato Y, Higuchi T, Yoshioka S, Maeda M, Ueda M, Fujii S: Human blastocysts and endometrial epithelial cells express activated leukocyte cell adhesion molecule (ALCAM/CD166). J Clin Endocrinol Metab 2003, 88:3437-3443

32. Haouzi D, Dechaud H, Assou S, Monzo C, de Vos J, Hamamah S: Transcriptome analysis reveals dialogues between human trophectoderm and endometrial cells during the implantation period. Hum Reprod 2011, 26:1440-1449

33. Abumaree MH, Al Jumah MA, Kalionis B, Jawdat D, Al Khaldi A, AlTalabani AA, Knawy BA: Phenotypic and functional characterization of mesenchymal stem cells from chorionic villi of human term placenta. Stem Cell Rev 2013, 9:16-31

34. Darcy KM, Zangani D, Wohlhueter AL, Huang RY, Vaughan MM, Russell JA, Ip MM: Changes in ErbB2 (her-2/neu), ErbB3, and ErbB4 during growth, differentiation, and apoptosis of normal rat mammary epithelial cells. J Histochem Cytochem 2000, 48:63-80

35. Klonisch T, Wolf P, Hombach-Klonisch S, Vogt S, Kuechenhoff A, Tetens F, Fischer B: Epidermal growth factor-like ligands and erbB genes in the peri-implantation rabbit uterus and blastocyst. Biol Reprod 2001, 64:1835-1844

36. Levy R: The role of apoptosis in preeclampsia. Isr Med Assoc J 2005, 7:178-181 